\title{
Semiparametric estimation of the non-mixture cure model with auxiliary survival information
}

\author{
Bo Han ${ }^{1}$, Ingrid Van Keilegom², and Xiaoguang Wang ${ }^{1, *}$ \\ ${ }^{1}$ School of Mathematical Sciences, Dalian University of Technology, Liaoning, China \\ ${ }^{2}$ Research Center for Operations Research and Statistics, KU Leuven, Leuven, Belgium \\ *email: wangxg@dlut.edu.cn \\ email: ingrid.vankeilegom@kuleuven.be
}

\begin{abstract}
Summary: With rapidly increasing data sources, statistical methods that make use of external information are gradually becoming popular tools in medical research. In this article, we efficiently synthesize the auxiliary survival information and propose a semiparametric estimation method for the combined empirical likelihood in the framework of the non-mixture cure model, to enhance inference about the associations between exposures and disease outcomes. The auxiliary survival probabilities from external sources are firstly summarized as unbiased estimation equations, which help produce more efficient estimates of the effects of interest and improve the prediction accuracy for the risk of the event. Then we develop a Bernstein-based sieve empirical likelihood method to estimate the parametric and nonparametric components simultaneously. Such an estimation procedure allows us to reduce the computation burden while preserving the shape constraint on the baseline distribution function. The resulting estimators for the true associations are strongly consistent and asymptotically normal. Instead of collecting substantial exposure data, the auxiliary survival information at multiple time points is incorporated in our framework, which further reduces the mean squared error of the estimators. This contributes to biomarker evaluation and treatment effect analysis within smaller studies in clinical trials. We show how to choose the number of auxiliary survival probabilities appropriately and provide a guideline for practical applications. Simulation studies demonstrate that the estimators enjoy large gains in efficiency. A melanoma dataset is analyzed for illustrating the methodology.
\end{abstract}

KEY WORDS: Biomarker evaluation; Empirical likelihood; Information synthesis; Non-mixture cure model; Sieve method.

This paper has been submitted for consideration for publication in Biometrics 


\section{Introduction}

Exploiting external information to enhance modeling and inference has attracted increasing attention in many disciplines. The empirical likelihood regression method (see e.g., Huang et al., 2016) ensures, by synthesizing summary information from big databases or existing papers, more efficient estimates of the associations of interest within smaller studies. The above method is capable of modeling individual-level interactions directly such as the treatmenteffect modifier, which can characterize the potential treatment effect heterogeneity and avoid selective reporting in primary studies.

A motivating example in this article is a melanoma study on aggregate data and individuallevel data. The aggregate data are extracted from the Surveillance, Epidemiology and End Results (SEER) database which gathers data from 18 cancer registries covering approximately $28 \%$ of the U.S. population. For the aggregate data of sample size 49,364, the results of the analysis in Section 6 show that tumor size and gender have main effects on the survival. However, these prognostic biomarkers are not identified when analyzing the random sampling individual-level data of sample size 300. On the other hand, although an individual-level analysis can provide the assessments of biomarkers and treatment effects, the evaluations may not be accurate in magnitude when the available data are limited. Thus it is of clinical value to develop statistical methods that make use of auxiliary information to enhance inference and address these issues.

In medical practice, lifetime data collected for successful disease management and treatment selection, frequently include a cure fraction. This is the case for certain cancers, where the 5-year relative survival probability, shown in Figure 1, is high for data from the SEER Program of the National Cancer Institute. The promotion time cure model, also named the non-mixture cure model, was proposed by Yakovlev and Tsodikov (1996) to take the cure feature into account. This class of models is an extension of the Cox proportional hazards 
model (Cox, 1972), by allowing for a cure fraction, and has a biological interpretation in the case of cancer relapse (see Chen et al., 1999). The nonparametric component in such models is required to be a proper baseline cumulative distribution function. To deal with this nonparametric component, a spline-based sieve maximum likelihood estimator was developed by $\mathrm{Hu}$ and Xiang (2013) for the non-mixture cure model. Portier et al. (2017) studied the asymptotic properties of the nonparametric maximum likelihood estimators under the non-mixture cure model, and developed a bootstrap procedure that allows for a consistent approximation of the asymptotic distribution of the estimators. We also refer to Amico and Van Keilegom (2018) for a recent review of cure models.

\section{[Figure 1 about here.]}

This article proposes a semiparametric estimation method for the non-mixture cure model with auxiliary information coming from external sources. The auxiliary information is formulated via unbiased estimating equations and is then combined with the likelihood function via the empirical likelihood method, see Owen (1988), Qin and Lawless (1994) and Molanes-Lopez et al. (2009). The constructed empirical likelihood involves an infinitedimensional function which is difficult to be profiled out of the likelihood function. In general, the infinite-dimensional parameter space is too large to obtain appropriate estimates. To overcome this difficulty, we utilize the Bernstein-based sieve method (Lorentz, 1986) to obtain estimators of the parametric and nonparametric components simultaneously. This approach is advantageous in controlling the expansion of the dimensionality of the model as the sample size increases. And such an approximation based on Bernstein polynomials enables the specific shape constraint over the entire support for the nonparametric component.

Inspired by the fact that the survival probabilities of subjects change over time, we formulate the subgroup survival probabilities at more than one survival time point, which promotes the utilization of the auxiliary survival information. Furthermore, by analyzing 
the overall trend of the mean squared error, we summarize the appropriate number of survival time points for small studies with different sample sizes, which is helpful for practical applications. The proposed semiparametric procedure with the auxiliary survival information at multiple survival time points can infer about the associations or differences of interest with enhanced efficiency.

The rest of the article is organized as follows. The non-mixture cure model and the formulation of the auxiliary survival information are given in Section 2. In Section 3, we construct the combined empirical likelihood and describe the estimation method. The asymptotic properties of the proposed estimators are given in Section 4. Simulation studies and data analyses are executed in Section 5 and Section 6. A discussion is presented in Section 7. The proofs, additional simulations and data analyses are provided in Web Appendices.

\section{Model and auxiliary survival information}

Let $T$ denote the event time of interest, let $\mathbf{Z} \in \mathbb{R}^{p}$ be a vector of associated covariates and let $S(t \mid \mathbf{Z})=P(T>t \mid \mathbf{Z})$ be the conditional survival function of $T$ given $\mathbf{Z}$. The non-mixture cure model can be formulated as follows:

$$
S(t \mid \mathbf{Z})=\exp \left\{-\eta\left(\mathbf{Z}^{T} \boldsymbol{\beta}\right) F(t)\right\}
$$

where $\eta(\cdot)$ is an increasing, known and positive link function, $\boldsymbol{\beta}$ is a $p$-dimensional vector of regression parameters, and $F(\cdot)$ is a completely unspecified baseline distribution function.

Model (1) allows for a nonzero cure rate as $t \rightarrow \infty$, i.e., $S(\infty \mid \mathbf{Z})=\exp \left\{-\eta\left(\mathbf{Z}^{T} \boldsymbol{\beta}\right)\right\}>0$, thus it is especially appealing when statistical studies primarily focus on the evaluation of risk factors in the presence of a cure fraction. With the conditional hazard function $h(t \mid \mathbf{Z})=\eta\left(\mathbf{Z}^{T} \boldsymbol{\beta}\right) f(t)$, where $f(t)=d F(t) / d t$, the non-mixture cure model preserves the proportional hazards structure. The link function usually takes the form $\eta\left(\mathbf{Z}^{T} \boldsymbol{\beta}\right)=\exp \left(\mathbf{Z}^{T} \boldsymbol{\beta}\right)$ and $\boldsymbol{\beta}$ includes an intercept $\beta_{0}$ to hold a flexible baseline cure rate $\exp \left\{-\exp \left(\beta_{0}\right)\right\}$. 
In follow-up studies, the event time of interest may be censored due to the termination of the study or loss to follow-up. Thus we observe the possibly censored survival time $Y=$ $\min \{T, C\}$ and $\delta=I(T \leqslant C)$, where $C$ is the censoring time and $\delta$ is the indicator of the event. Denote the observation set by $\mathcal{O}=\left\{\mathbf{O}_{i}: \mathbf{O}_{i}=\left(Y_{i}, \delta_{i}, \mathbf{Z}_{i}\right), i=1, \ldots, n\right\}$ consisting of $n$ independent and identically distributed (i.i.d.) copies of $(Y, \delta, \mathbf{Z})$. It is assumed throughout that the event time $T$ is independent of the censoring time $C$ conditional on $\mathbf{Z}$, which is a widely used assumption in biometrical studies.

To improve the estimation of the parameters in the model, we introduce survival probabilities for $M$ subgroups as auxiliary survival information. More precisely, the auxiliary survival information can be formulated in the form $P\left(T>t_{k}^{*} \mid \mathbf{Z} \in \boldsymbol{\Omega}_{m}\right)=\phi_{k m}, k=1, \ldots, K, m=$ $1, \ldots, M$, where $t_{k}^{*}$ is the prespecified $k$-th time point $(k=1, \ldots, K)$, and $\Omega_{m}$ denotes the $m$-th subgroup $(m=1, \ldots, M)$, whose $t_{k}^{*}$-year survival probability $\phi_{k m}$ can be obtained from external sources. By the definition of $\phi_{k m}$, it is feasible that the covariates are not completely comparable across the aggregate data and the individual-level data. More details are presented in the simulation section and the data analysis section.

Employing the double expectation formula, we have $P\left(T>t_{k}^{*}, \mathbf{Z} \in \boldsymbol{\Omega}_{m}\right)-\phi_{k m} P(\mathbf{Z} \in$ $\left.\boldsymbol{\Omega}_{m}\right)=E\left[E\left\{I\left(T>t_{k}^{*}\right) I\left(\mathbf{Z} \in \boldsymbol{\Omega}_{m}\right)-\phi_{k m} I\left(\mathbf{Z} \in \boldsymbol{\Omega}_{m}\right) \mid \mathbf{Z}\right\}\right]=0$. Under model (1), it follows that $E\left(I\left(\mathbf{Z} \in \boldsymbol{\Omega}_{m}\right)\left[\exp \left\{-\eta\left(\mathbf{Z}^{T} \boldsymbol{\beta}\right) F\left(t_{k}^{*}\right)\right\}-\phi_{k m}\right]\right)=0$. We consider the baseline distribution function evaluated at $t_{k}^{*}$ as a new parameter and denote it by $\alpha_{k}=F\left(t_{k}^{*}\right)$. Then the estimating function can be written as $\Psi_{k m}\left(\mathbf{Z}, \boldsymbol{\beta}, \alpha_{k}\right):=I\left(\mathbf{Z} \in \boldsymbol{\Omega}_{m}\right)\left[\exp \left\{-\eta\left(\mathbf{Z}^{T} \boldsymbol{\beta}\right) \alpha_{k}\right\}-\phi_{k m}\right]$. Therefore, the subgroup survival probabilities under multiple survival time points satisfy the equations $E\left\{\Psi_{k m}\left(\mathbf{Z}, \boldsymbol{\beta}, \alpha_{k}\right)\right\}=0, k=1, \ldots, K, m=1, \ldots, M$

\section{Estimation}

In this section, we construct the combined empirical likelihood with the auxiliary survival information and describe the semiparametric estimation procedure. For the non-mixture cure 
model (1), the full log-likelihood function based on the observed data is given by

$$
l_{n}(\boldsymbol{\beta}, F(\cdot), G(\cdot))=\sum_{i=1}^{n}\left[\delta_{i} \log \left\{\eta\left(\mathbf{Z}_{i}^{T} \boldsymbol{\beta}\right) f\left(Y_{i}\right)\right\}-\eta\left(\mathbf{Z}_{i}^{T} \boldsymbol{\beta}\right) F\left(Y_{i}\right)\right]+\sum_{i=1}^{n} \log \left\{d G\left(\mathbf{Z}_{i}\right)\right\},
$$

where $G(\cdot)$ is the distribution function of $\mathbf{Z}$.

Next, the method of empirical likelihood is employed to synthesize the auxiliary survival information. The key is to formulate the auxiliary survival information in the form of unbiased estimating equations. Consider the following maximization problem:

$$
\max _{\left\{p_{i}\right\}_{i=1}^{n}} \sum_{i=1}^{n} \log p_{i}, \quad \text { such that } \quad 0 \leqslant p_{i} \leqslant 1, \quad \sum_{i=1}^{n} p_{i}=1, \quad \sum_{i=1}^{n} p_{i} \Psi\left(\mathbf{Z}_{i}, \boldsymbol{\beta}, \boldsymbol{\alpha}\right)=\mathbf{0}
$$

where $p_{i}$ is the jump of $G(\cdot)$ at $\mathbf{Z}_{i}$ and $\boldsymbol{\Psi}\left(\mathbf{Z}_{i}, \boldsymbol{\beta}, \boldsymbol{\alpha}\right)=\left(\Psi_{11}\left(\mathbf{Z}_{i}, \boldsymbol{\beta}, \alpha_{1}\right), \ldots, \Psi_{K M}\left(\mathbf{Z}_{i}, \boldsymbol{\beta}, \alpha_{K}\right)\right)^{T}$. By the Lagrange multiplier method, the solution of this problem is given by $p_{i}=n^{-1}\{1+$ $\left.\boldsymbol{\xi}^{T} \boldsymbol{\Psi}\left(\mathbf{Z}_{i}, \boldsymbol{\beta}, \boldsymbol{\alpha}\right)\right\}^{-1}$, where the Lagrange multiplier vector $\boldsymbol{\xi}=\left(\xi_{11}, \ldots, \xi_{K M}\right)^{T}$ is the solution of $n^{-1} \sum_{i=1}^{n} \boldsymbol{\Psi}\left(\mathbf{Z}_{i}, \boldsymbol{\beta}, \boldsymbol{\alpha}\right)\left\{1+\boldsymbol{\xi}^{T} \boldsymbol{\Psi}\left(\mathbf{Z}_{i}, \boldsymbol{\beta}, \boldsymbol{\alpha}\right)\right\}^{-1}=\mathbf{0}$. By incorporating the auxiliary survival probabilities, the combined empirical log-likelihood function can therefore be written as

$$
l_{n}(\boldsymbol{\theta})=\sum_{i=1}^{n}\left[\delta_{i} \log \left\{\eta\left(\mathbf{Z}_{i}^{T} \boldsymbol{\beta}\right) f\left(Y_{i}\right)\right\}-\eta\left(\mathbf{Z}_{i}^{T} \boldsymbol{\beta}\right) F\left(Y_{i}\right)\right]-\sum_{i=1}^{n} \log \left\{1+\boldsymbol{\xi}^{T} \boldsymbol{\Psi}\left(\mathbf{Z}_{i}, \boldsymbol{\beta}, \boldsymbol{\alpha}\right)\right\},
$$

where $\boldsymbol{\theta}=\left(\boldsymbol{\beta}^{T}, \boldsymbol{\alpha}^{T}, \boldsymbol{\xi}^{T}, F\right)^{T}$. Note that the parameter vector $\boldsymbol{\theta}$ includes the infinite-dimensional parameter $F(\cdot)$. Since the infinite-dimensional parameter space is too large to obtain reasonable estimates, in the following, we develop the sieve method to tackle this difficulty.

Let $\|\cdot\|$ be the Euclidean norm and denote the parameter space of $\boldsymbol{\theta}$ by

$$
\boldsymbol{\Theta}=\left\{\boldsymbol{\theta}=(\boldsymbol{\iota}, F)^{T}: \iota=\left(\boldsymbol{\beta}^{T}, \boldsymbol{\alpha}^{T}, \boldsymbol{\xi}^{T}\right)^{T} \in \mathcal{B}, F \in \Gamma\right\}=\mathcal{B} \times \Gamma,
$$

where $\mathcal{B}=\left\{\boldsymbol{\iota}: \iota \in \mathbb{R}^{p+K+K \times M},\|\boldsymbol{\iota}\| \leqslant L, 0 \leqslant \alpha_{k} \leqslant 1, k=1, \ldots, K\right.$, and $\alpha_{l} \leqslant \alpha_{k}$ if $\left.t_{l}^{*} \leqslant t_{k}^{*}\right\}$, $\Gamma=\left\{F: F \in C^{m}[0, \tau],\left|F^{(m)}\left(t_{1}\right)-F^{(m)}\left(t_{2}\right)\right| \leqslant C\left|t_{1}-t_{2}\right|^{a}, \forall t_{1}, t_{2} \in[0, \tau]\right\}$ for constants $L, C \in(0, \infty), a \in(0,1]$, a nonnegative integer $m$ with $r=m+a>0.5$ and $\tau=\inf \{t$ : $F(t)=1\}$, which is supposed to be finite. Such a smoothness assumption for $F(t)$ is often adopted in semiparametric estimation settings. We define the set $\boldsymbol{\Theta}_{n}=\mathcal{B} \times \Gamma_{n}$, where

$$
\Gamma_{n}=\left\{F_{n}: F_{n}(t)=\sum_{i=0}^{N} \gamma_{i} b_{i, N}(t), 0 \leqslant \gamma_{0} \leqslant \cdots \leqslant \gamma_{N}=1, \sum_{i=0}^{N} \gamma_{i} \leqslant M_{n}, t \in[0, \tau]\right\}
$$


with $b_{i, N}(t)$ being the Bernstein basis function of order $N=o\left(n^{\nu}\right)$ for some $\nu \in(0,1)$, $b_{i, N}(t)=\left(\begin{array}{c}N \\ i\end{array}\right)\left(\frac{t}{\tau}\right)^{i}\left(1-\frac{t}{\tau}\right)^{N-i}$ and $M_{n}=O\left(n^{\varsigma}\right)$ for some $\varsigma \in(0, \infty)$ controlling the size of $\boldsymbol{\Theta}_{n}$. Note that the constraint $\gamma_{0} \leqslant \cdots \leqslant \gamma_{N}$ ensures that the baseline distribution function $F_{n}(\cdot)$ is non-decreasing (Babu et al., 2002 and Wang and Ghosh, 2012).

Let $\|g(X)\|_{p}=\left(\int|g(x)|^{p} d P\right)^{1 / p}$ denote the $L_{p}(P)$-norm of a function $g(\cdot)$ with $X$ being distributed according to the probability measure $P$. For any $\boldsymbol{\theta}_{1}, \boldsymbol{\theta}_{2} \in \boldsymbol{\Theta}$, we define the distance $d\left(\boldsymbol{\theta}_{1}, \boldsymbol{\theta}_{2}\right)=\left\{\left\|\boldsymbol{\iota}_{1}-\boldsymbol{\iota}_{2}\right\|^{2}+\left\|F_{1}-F_{2}\right\|_{2}^{2}\right\}^{1 / 2}$. By Theoreom 1.1.1 in Lorentz (1986), for any $\boldsymbol{\theta} \in \boldsymbol{\Theta}$, there exists $\pi_{n} \boldsymbol{\theta}=\left(\boldsymbol{\iota}, F_{n}\right) \in \boldsymbol{\Theta}_{n}$ such that $d\left(\boldsymbol{\theta}, \pi_{n} \boldsymbol{\theta}\right) \leqslant C\left\|F-F_{n}\right\|_{\infty} \rightarrow 0$ as $n \rightarrow \infty$. It implies that $\boldsymbol{\Theta}_{n}$ can be regarded as the sieve space to approximate $\Theta$ as $n \rightarrow \infty$. Therefore, we can obtain an estimator via

$$
\widehat{\boldsymbol{\theta}}_{n}=\underset{\boldsymbol{\theta} \in \Theta_{n}}{\arg \max } l_{n}(\boldsymbol{\theta}) .
$$

In the computation of $\widehat{\boldsymbol{\theta}}_{n}$, the Quasi-Newton algorithm can be employed for the optimization of $l_{n}\left(\boldsymbol{\iota}, \gamma_{0}, \ldots, \gamma_{N}\right)$ subject to $0 \leqslant \alpha_{k} \leqslant 1, \alpha_{l} \leqslant \alpha_{k}$ if $t_{l}^{*} \leqslant t_{k}^{*}$, and $0 \leqslant \gamma_{0} \leqslant \cdots \leqslant \gamma_{N}=1$ (where we identify $F_{n}$ with $\gamma_{0}, \ldots, \gamma_{N}$ ). The optimization can be implemented via the maxLik package in the software R. Compared with the nonparametric maximum likelihood estimator of $F(\cdot)$, the estimation based on the sieve method can significantly reduce the computational cost since the order $N$ grows much slower than $O(n)$.

The proposed estimation method involves the choice of the order $N$. The AIC considers a trade-off between the goodness-of-fit and the simplicity of the model. When selecting a finite dimensional approximating model, the AIC is asymptotically efficient and widely adopted (Hurvich and Tsai, 1989; Xue et al., 2004; Geerdens et al., 2013). The order $N$ can be determined by minimizing the AIC given by $\mathrm{AIC}=-2 l_{n}\left(\widehat{\boldsymbol{\theta}}_{n}\right)+2(\widetilde{p}+N+1)$, where $\widetilde{p}=p+K+K \times M$.

REMARK 1: A crucial assumption for the proposed method is that the data distribution is the same for the individual-level data and for the population that generates the auxiliary 
information. When this assumption is violated, Chatterjee et al. (2016) proposed the synthetic maximum likelihood estimation for regression models to allow heterogeneity between the individual-level and external covariate distributions. The empirical likelihood-based approach was developed by Han and Lawless (2019) to increase the efficiency of estimation for the case of heterogeneity. Following the line of Han and Lawless (2019), the estimation procedure for the non-mixture cure model is developed to allow heterogeneity in Web Appendix D.

REMARK 2: When the external data set is not very large, the uncertainty in the auxiliary information is non-negligible and affects the asymptotic behavior of the estimators (see e.g., Chatterjee et al., 2016; Han and Lawless, 2019; Huang and Qin, 2020). We explicitly show the effects of the uncertainty on the representation result and on the asymptotic variances of the proposed estimators in Web Appendix D.

\section{Asymptotic properties}

In this section, we present the asymptotic properties of the estimator $\widehat{\boldsymbol{\theta}}_{n}$. Denote the true parameter by $\boldsymbol{\theta}_{0}=\left(\boldsymbol{\beta}_{0}^{T}, \boldsymbol{\alpha}_{0}^{T}, \boldsymbol{\xi}_{0}^{T}, F_{0}\right)^{T}$. The regularity conditions needed for the asymptotic properties are as follows:

(C1) (i) The covariate $\mathbf{Z}$ is bounded with probability one. (ii) If there exists a vector $\mathbf{b} \in \mathbb{R}^{p}$ such that $P\left(\mathbf{Z}^{T} \mathbf{b}=0\right)=1$, then $\mathbf{b}=\mathbf{0}$. (iii) The link function $\eta(\cdot)$ is a strictly increasing function and $\eta(\cdot)>0$.

(C2) The true $\boldsymbol{\iota}_{0}$ is an interior point of $\mathcal{B}$, and the true baseline distribution function $F_{0}(\cdot)$ is an interior point of $\Gamma$.

(C3) Conditional on $\mathbf{Z}$, the censoring time $C$ is independent of the survival time $T$. Both $C$ and $T$ are absolutely continuous. Moreover, $T>\tau$ implies that $T=+\infty$, and $P(C>$ $\tau \mid \mathbf{Z})>0$ a.s. 
(C4) The function $\vartheta(\boldsymbol{\theta})$ is continuously differentiable and $\left\|\dot{\vartheta}\left(\boldsymbol{\theta}_{0}\right)\right\|<\infty$, where $\vartheta(\boldsymbol{\theta})$ and $\left\|\dot{\vartheta}\left(\boldsymbol{\theta}_{0}\right)\right\|$ are defined in equations (1) and (2) of Web Appendix C.

Conditions (C1)-(ii,iii) and (C3) ensure the identifiability of the non-mixture cure model (Portier et al., 2017). Condition (C2) indicates that $\boldsymbol{\theta}_{0}$ is an interior point of $\boldsymbol{\Theta}$. Condition (C4) is utilized to prove the asymptotic normality.

Theorem 1 (Strong Consistency): Suppose Conditions (C1)-(C3) hold, then we have

$$
\left\|\widehat{\boldsymbol{\beta}}_{n}-\boldsymbol{\beta}_{0}\right\| \rightarrow 0 \text { and }\left\|\widehat{F}_{n}-F_{0}\right\|_{2} \rightarrow 0
$$

almost surely.

We sketch the key steps of the proof of Theorem 1. Let $l(\boldsymbol{\theta} ; \mathbf{O})=\delta \log \left\{\eta\left(\mathbf{Z}^{T} \boldsymbol{\beta}\right) f(Y)\right\}-$ $\eta\left(\mathbf{Z}^{T} \boldsymbol{\beta}\right) F(Y)-\log \left\{1+\boldsymbol{\xi}^{T} \mathbf{\Psi}(\mathbf{Z}, \boldsymbol{\beta}, \boldsymbol{\alpha})\right\}$ and $\mathbf{O}=(Y, \delta, \mathbf{Z})$. Denote $P_{n} l(\boldsymbol{\theta} ; \mathbf{O}):=n^{-1} \sum_{i=1}^{n} l\left(\boldsymbol{\theta} ; \mathbf{O}_{i}\right)$ and $P l(\boldsymbol{\theta} ; \mathbf{O}):=E l(\boldsymbol{\theta} ; \mathbf{O})$. We show that $\sup _{\boldsymbol{\theta} \in \boldsymbol{\Theta}_{n}}\left|P_{n} l(\boldsymbol{\theta} ; \mathbf{O})-P l(\boldsymbol{\theta} ; \mathbf{O})\right| \rightarrow 0$ almost surely. Next, utilizing this property, we demonstrate that $\left|P l\left(\widehat{\boldsymbol{\theta}}_{n} ; \mathbf{O}\right)-\operatorname{Pl}\left(\boldsymbol{\theta}_{0} ; \mathbf{O}\right)\right| \rightarrow 0$ almost surely. By the relationship between the Kullback-Leibler information and the Hellinger metric, we then conclude that $\widehat{\boldsymbol{\theta}}_{n}$ converges to $\boldsymbol{\theta}_{0}$ almost surely, which leads to Theorem 1.

Theorem 2 (Rate of Convergence): Suppose Conditions (C1)-(C3) hold, $r \in[1, \infty)$ and $\nu \in(0,1)$, then we have

$$
\left\|\widehat{\boldsymbol{\beta}}_{n}-\boldsymbol{\beta}_{0}\right\|=O_{p}\left(n^{-\min (r \nu / 2,(1-\nu) / 2)}\right), \quad\left\|\widehat{F}_{n}-F_{0}\right\|_{2}=O_{p}\left(n^{-\min (r \nu / 2,(1-\nu) / 2)}\right) .
$$

This rate is optimal if $r \nu=1-\nu$, i.e. when $\nu=1 /(1+r)$, in which case the rate of convergence is $O\left(n^{-r /\{2(1+r)\}}\right)$. This implies that the convergence rate is $n^{-1 / 3}$ if $r=2$ and tends to $n^{-1 / 2}$ as $r$ increases. In the next theorem, we further conclude that $\widehat{\boldsymbol{\beta}}_{n}$ can achieve the $n^{-1 / 2}$ convergence rate. The rate of convergence in Theorem 2 is utilized to derive the asymptotic normality of the estimator.

Theorem 3 (Asymptotic Normality): Suppose Conditions (C1)-(C4) hold, $r \in(2, \infty)$ 
and $\nu \in(1 / 2 r, 1 / 3)$, then we have

$$
\sqrt{n}\left[\boldsymbol{\mu}_{1}^{T}\left(\widehat{\boldsymbol{\beta}}_{n}-\boldsymbol{\beta}_{0}\right)+\int_{0}^{\tau} u(t)\left\{\widehat{F}_{n}(t)-F_{0}(t)\right\} d t\right] \rightarrow N\left(0,\left\|\dot{\vartheta}_{\boldsymbol{\beta}, F}\left(\boldsymbol{\theta}_{0}\right)\right\|^{2}\right)
$$

in distribution, where $\boldsymbol{\mu}_{1} \in \mathbb{R}^{p}, u \in \Gamma$ and $\left\|\dot{\vartheta}_{\boldsymbol{\beta}, F}\left(\boldsymbol{\theta}_{0}\right)\right\|$ is given in equation (4) of Web Appendix $C$.

Theorem 3 is quite general and allows us to make inference about the regression parameters. For example, with $u(\cdot) \equiv 0$, we obtain that $\sqrt{n} \boldsymbol{\mu}_{1}^{T}\left(\widehat{\boldsymbol{\beta}}_{n}-\boldsymbol{\beta}_{0}\right) \rightarrow N\left(0,\left\|\dot{\vartheta}_{\boldsymbol{\beta}}\left(\boldsymbol{\theta}_{0}\right)\right\|^{2}\right)$ in distribution, where $\left\|\dot{\vartheta}_{\boldsymbol{\beta}}\left(\boldsymbol{\theta}_{0}\right)\right\|$ is given in equation (5) of Web Appendix C. Then applying the Cramér-Wold theorem, we obtain that $\widehat{\boldsymbol{\beta}}_{n}$ is asymptotically normal. The proofs for Theorem 1-3 are provided in Web Appendix C.

It is difficult to present an explicit expression for the asymptotic variance of $\widehat{\boldsymbol{\beta}}$. Alternatively, we adopt the nonparametric bootstrap method to estimate the asymptotic variance, which is easy to implement and works well in simulation results.

\section{Simulation studies}

In this section, we carry out simulation studies to assess the performance of the proposed estimation procedure. The first scenario involves discrete and continuous random variables to mimic clinical treatments and potential risk factors. The treatment heterogeneity is of clinical value in tailored therapies. Thus an interaction between discrete and continuous random variables, representing a treatment-by-covariate interaction, is considered in the second scenario. The two scenarios are formulated as

$$
\begin{aligned}
& \text { Scenario I : } S\left(t \mid Z_{1}, Z_{2}\right)=\exp \left\{-\exp \left(\beta_{0}+\beta_{1} Z_{1}+\beta_{2} Z_{2}\right) F_{0}(t)\right\} \\
& \text { Scenario II : } S\left(t \mid Z_{1}, Z_{2}\right)=\exp \left\{-\exp \left(\beta_{0}+\beta_{1} Z_{1}+\beta_{2} Z_{2}+\beta_{3} Z_{1} Z_{2}\right) F_{0}(t)\right\} .
\end{aligned}
$$

For both scenarios the true values of the parameters are $\beta_{0}=0.5$ or $-1, \beta_{1}=\beta_{2}=\beta_{3}=1$, $F_{0}(t)=\left(1-e^{-2 t}\right) /\left(1-e^{-3}\right) I(0 \leqslant t \leqslant 1.5)$, and $Z_{1} \sim \operatorname{Bernoulli}(0.5), Z_{2} \sim U(-1,1)$, $C \sim U\left(0, c_{0}\right)$, where $c_{0}$ is used to control the censoring rate. Note that $\tau=1.5$ and the 
settings where $\beta_{0}=0.5$ and $\beta_{0}=-1$ correspond to a low-level cure rate and a moderatelevel cure rate, respectively.

First, we consider the relationship between the MSE and the number of auxiliary survival time points $K$. Set the subgroups and their auxiliary survival time by $\Omega_{1}=\left\{\left(Z_{1}, Z_{2}\right): Z_{1}=\right.$ $0\}, \boldsymbol{\Omega}_{2}=\left\{\left(Z_{1}, Z_{2}\right): Z_{1}=1\right\}, \boldsymbol{t}^{*}=\left\{t_{k}^{*}\right\}_{k=1}^{K}$, where $t_{k}^{*}=k \tau /(K+1)$ and the subgroups $\boldsymbol{\Omega}_{1}$ and $\Omega_{2}$ mimic the groups of subjects receiving different clinical treatments. The results for the MSE are based on $n=200,400$ and 600 with 400 replications.

Before calculating the estimators of $\boldsymbol{\beta}_{0}$, we need to determine the optimal value of $N$, for which we minimize the AIC given in Section 3. Consider Scenario I with $\boldsymbol{\beta}_{0}=(-1,1,1)$ and $n=200$ for example. The results for the AIC are presented in Web Figure 1, which shows that the optimal value of $N$ is $N=4$ in this setting.

To select an appropriate value of $K$, we calculate the mean squared error (MSE) given by $\operatorname{MSE}(K)=E\left\{\left(\widehat{\boldsymbol{\beta}}_{n, K}-\boldsymbol{\beta}_{0}\right)^{T} V\left(\widehat{\boldsymbol{\beta}}_{n, K}-\boldsymbol{\beta}_{0}\right)\right\}$, where $\widehat{\boldsymbol{\beta}}_{n, K}$ denotes the estimator obtained by using the subgroup survival probabilities at $\boldsymbol{t}^{*}=\left\{t_{1}^{*}, \ldots, t_{K}^{*}\right\}$, and $V$ is the covariance matrix of the covariates. In Web Figure 2 we provide the results of the MSE. The results suggest that an appropriate value of $K$ is 4 for the individual-level data with $n \leqslant 400$. When the sample size is larger than 400 , we can choose $K=3$ or even smaller.

To further assess the performance of the estimation method, we execute Scenario I and Scenario II for samples of size $n=200$ and 400 with 400 replications. The standard errors of the estimated regression coefficients are computed by the nonparametric bootstrap method with 100 bootstrap samples. The following summary statistics are considered: the empirical bias (Bias), the sample standard deviation (SD), the average of the estimated standard errors (SE), the $95 \%$ empirical coverage probability (CP) and the relative efficiency (RE), defined as the ratio of the two estimated variances.

In the summary tables (Tables 1 and 2), ML represents the sieve maximum likelihood 
estimator without the auxiliary information, and EL means the proposed semiparametric estimator obtained using the auxiliary survival information. In particular, EL1 indicates that the survival probabilities of the two subgroups at $t^{*}=0.75$ are accommodated into the estimation procedure. EL2 corresponds to the proposed method with the subgroup survival probabilities at the survival time $\boldsymbol{t}^{*}=\left\{t_{1}^{*}, t_{2}^{*}\right\}=\{0.375,1.125\}$. Similarly, EL4 stands for the proposed method with $\boldsymbol{t}^{*}=\left\{t_{1}^{*}, \ldots, t_{4}^{*}\right\}=\{0.3,0.6,0.9,1.2\}$.

[Table 1 about here.]

The survival probabilities of the two subgroups under single and multiple time points are displayed in Web Table 1 for the two scenarios. Table 1 reports the summarized results for Scenario I for the two cure rates. The results show that the estimators are asymptotically unbiased. The SEs agree well with the SDs, and the CPs are close to the nominal level of $95 \%$. Compared with the estimator obtained by the ML method, the proposed estimator realizes a substantial gain in efficiency. For the moderate-level cure rate setting, under $n=200$, the REs for the EL1 method range from 2.3 to 4.2 while the REs for the EL4 method range from 7.7 to 13.0. Thus, the estimation procedure with the auxiliary survival probabilities under multiple time points enjoys a higher efficiency gain than that under a single time point.

[Table 2 about here.]

Table 2 presents the summarized results for Scenario II for the two cure rates. The results in Table 2 show that the proposed estimator accomplishes an important efficiency gain for the interaction. This suggests that the proposed method is an important statistical tool in the identification of significant interactions. It is well known that the significance of $\beta_{3}$ is valued in subgroup identification, which would suggest the presence of treatment heterogeneity.

[Figure 2 about here.]

In order to study further the efficiency gain under Scenarios I and II, we graphically 
present the estimates of $\boldsymbol{\beta}$ under the different estimation methods by means of boxplots. The estimates of $\boldsymbol{\beta}$ for the ML and EL methods are shown in Figure 2, under the moderatelevel cure rate of Scenario I and $n=400$, where the horizontal dashed line means the true parameter value. Compared with the ML method, the EL method enjoys a lower variability of the estimates especially for EL4.

[Figure 3 about here.]

In addition, Figure 3 presents the estimates of the nonparametric component $F(\cdot)$ under the moderate-level cure rate of Scenario I and $n=400$, over 400 simulated datasets (grey lines) under the different estimation methods, where the dashed line represents the average of the estimators. Similarly as before, the variability of the estimates under the EL method is smaller than that under the ML method. As more auxiliary survival information is considered in the combined empirical likelihood, the efficiency can be further improved for the estimator of the nonparametric component.

Next, we consider a case in which the aggregate data have fewer covariates than the individual-level data. Both datasets are generated from the model of Scenario I, but $Z_{2}$ is excluded from the aggregate data. That is, the covariates are $Z_{1}$ and $Z_{2}$ for the individuallevel data, and only $Z_{1}$ for the aggregate data. The values of the parameters are the same as for Scenario I. Web Table 2 presents the subgroup survival probabilities, while Web Table 3 reports the summarized regression results, revealing that the estimators are almost unbiased and enjoy a substantial gain in efficiency.

\section{Data Example: Melanoma Study}

We now analyze survival data from a melanoma study, and evaluate the association between the time from diagnosis of melanoma until death of melanoma and a set of covariates. We consider the patients diagnosed with melanoma between 2008 and 2014 as the study 
population. The complete dataset is available from the SEER database. Especially, we select the database 'Incidence - SEER 18 Regs Research Data', the 'summary stage 2000(1998+)local, regional, distant' and 'Coding CS Tumor Size 001-988', where the code 001-988 denotes the exact size in millimeters. In the same spirit as Huang et al. (2016), we randomly select 300 cases from the complete dataset as the individual-level dataset for our analysis.

In this data analysis, the auxiliary survival information is obtained from the complete dataset. In fact, for an individual-level dataset from a small study, one may combine the auxiliary survival information from published articles, reports or other sources, and then utilize the proposed method to enhance inference about the associations of interest.

For the 49,364 cases in the complete dataset, the survival time ranges from 0 to 95 months with median 39, and men account for $60.0 \%$. The minimum, median and maximum of tumor sizes are $1 \mathrm{~mm}, 10 \mathrm{~mm}$ and $988 \mathrm{~mm}$, respectively. For these cases in the individual-level dataset, the range of the survival time in months is the same as that in the complete dataset. The median is 42 months and the percentage of men is $59.3 \%$. The censoring rates for the complete and individual-level datasets are $92.3 \%$ and $92.7 \%$, respectively.

We begin with an exploratory data analysis for this melanoma study. On one hand, the Kaplan-Meier (KM) estimates of the survival functions for the aggregate data by tumor size and gender are shown in Web Figure 3. The results suggest that tumor size and gender may have significant effects on survival.

On the other hand, for the individual-level data, we show the KM estimates by tumor size and gender in Web Figure 4. The estimated survival functions, by tumor size and gender, are relatively close for the individual-level study. For tumor size, the $p$-value for the log-rank test is less than 0.0001 for the aggregate data while it is $p=0.70$ for the individual-level data. For gender, we obtain that $p<0.0001$ for the aggregate data and $p=0.90$ for the individual-level data. This means that some risk factors may not be detected in the individual-level analysis 
due to the limited amount of data, which could lead to misleading conclusions. Instead of utilizing all the aggregate data, we combine the individual-level data with the auxiliary survival information from the aggregate data to precisely infer about the association in the smaller individual-level study.

We employ the proposed estimation procedure for the melanoma study. The KM estimate for all cases in the individual-level dataset is shown in Web Figure 5. The clear plateau in the right tail indicates that the survival data may include a cure fraction. Furthermore, the $p$-value of the nonparametric test in Maller and Zhou (1992) is less than 0.001 for the individual-level dataset. It means that this dataset satisfies the condition of Theorem 1 in Maller and Zhou (1992). Then adopting the method in Maller and Zhou (1992), a consistent estimate of the cure rate for this individual-level dataset is $90.0 \%$, and the confidence interval is $[86.0 \%, 94.2 \%]$. It indicates that cure models can be applied to this dataset.

We adopt the non-mixture cure model to analyze the dataset with the proposed method. Three covariates in the individual-level data are considered: gender, with 1 indicating male; age (normalized); tumor size, with 1 indicating more than $4 \mathrm{~mm}$ in size. The covariate in the complete dataset only contains gender, which implies that the covariates are not completely comparable across the two datasets. The ML and EL method are employed to estimate the parameters, and we calculate the variance of the parameter estimates via the nonparametric bootstrap method. By the discussion of the simulation results, an appropriate value of $K$ could be 4 for such an individual-level dataset of sample size 300 .

For the auxiliary survival probability, when $K=1$, we choose the 5 -year survival probability, since this is a widely adopted cancer statistic in reports of SEER to the nation on the status of cancer. When $K=4$, we choose the survival probabilities after 1 year, 2.5 years, 5 years and 7.5 years to utilize the auxiliary survival information uniformly. For the EL1 method, the subgroup survival probabilities at $t^{*}=60$ months are $92.8 \%$ 
for women (the subgroup $\Omega_{1}$ ) and $88.2 \%$ for men (the subgroup $\boldsymbol{\Omega}_{2}$ ), respectively. For the EL4 method, the subgroup survival probabilities at $\boldsymbol{t}^{*}=\{12,30,60,90\}$ for women and men are $\{98.2 \%, 95.3 \%, 92.8 \%, 91.6 \%\}$ and $\{97.1 \%, 92.5 \%, 88.2 \%, 86.1 \%\}$, respectively. These subgroup survival probabilities are obtained from the Kaplan-Meier estimates based on the corresponding subgroups of the complete dataset.

[Table 3 about here.]

Table 3 reports the estimated regression results for the melanoma study. The complete dataset is available in this data example and the regression results of the complete dataset are reported as a reference. As can be seen from Table 3, the proposed EL method can gain efficiency compared with the ML method. It means that the EL method can produce more accurate estimates for the regression coefficients. By the ML method, the estimated regression parameters are significant for age, gender and tumor size when using the complete dataset, while not significant for gender and tumor size when using the individual-level dataset. However, using the individual-level dataset, all estimators of the regression parameters are significant using the EL method. In other words, the proposed empirical likelihood regression analysis helps to identify the prognostic biomarkers, and accurately measure the effects of disease biomarkers on the outcome within a small study. These results highlight the clinical value of utilizing such a more efficient estimation procedure in cancer research.

We consider a case in which the covariates are transformed in different ways across the two datasets. The covariates of the individual-level data include gender, a binary variable for age with 1 indicating age less than 60, and tumor size. However, the covariate of the aggregate data only includes age as a continuous variable. In this case, age should be transformed to a discrete variable, which leads to the common subgroups $\Omega_{1}$ (those that are younger than 60 ) and $\Omega_{2}$ (those that are 60 or older). Using the Kaplan-Meier estimates, the estimated subgroup survival probabilities at $\boldsymbol{t}^{*}=\{12,30,60,90\}$ are $\{98.4 \%, 95.8 \%, 93.2 \%, 91.8 \%\}$ for 
$\Omega_{1}$ and $\{96.9 \%, 92.0 \%, 87.7 \%, 85.7 \%\}$ for $\Omega_{2}$, respectively. Web Table 4 reports the regression results. As expected, the proposed estimators realize gains in efficiency.

\section{Discussion}

In this article, we combined individual-level data with subgroup survival probabilities at different time points to estimate a non-mixture cure model. We constructed the combined empirical likelihood function by incorporating the $\boldsymbol{t}^{*}$-year subgroup survival probabilities in the likelihood. For approximating the nonparametric component, the sieve method based on Bernstein polynomials was utilized, which preserves the shape constraint on the baseline distribution function and reduces the computational burden. The asymptotic properties of the estimator were provided. The efficiency gains of the proposed procedure were demonstrated by extensive simulations and the data analysis of the melanoma study.

Approaches to exploit auxiliary information are widely developed in the literature (see e.g., Huang et al., 2016; Sheng et al., 2020). This article considered a topic of synthesizing auxiliary information in the framework of cure models. We regarded the databases of disease registries as the sources of auxiliary information, and utilize such information to provide more efficient estimates within smaller studies. Simulation results showed an efficiency gain in the estimated coefficients. The proposed estimation procedure, as the data analysis demonstrated, provided a more accurate evaluation of the associations of interest.

The combined empirical log-likelihood function reveals that the individual-level data and the auxiliary survival information make equal contributions to the likelihood. An extension is to allow for varying contributions in the likelihood (Hjort et al., 2018). Specifically, a hybrid likelihood function can be defined by $H_{n}=\prod_{i=1}^{n}\left\{h\left(Y_{i} \mid \mathbf{Z}_{i}\right)^{\delta_{i}} S\left(Y_{i} \mid \mathbf{Z}_{i}\right)\right\}^{1-w}\left\{d G\left(\mathbf{Z}_{i}\right)\right\}^{w}$, where $w \in[0,1 / 2]$ is a given balance parameter. Following the same line, after incorporating the subgroup survival probabilities, the sieve maximum likelihood estimation procedure can be adopted to estimate the non-mixture cure model. 
In real applications, it is possible that the auxiliary survival information from external sources is not consistent with the individual-level data. To test the conformity of the auxiliary survival information, the empirical likelihood ratio test statistic $R=2\left\{\sup _{\boldsymbol{\beta}, \boldsymbol{\alpha}, \boldsymbol{\xi}, F} l_{n}(\boldsymbol{\beta}, \boldsymbol{\alpha}, \boldsymbol{\xi}, F)-\right.$ $\left.\sup _{\boldsymbol{\beta}, \boldsymbol{\alpha}, F} l_{n}(\boldsymbol{\beta}, \boldsymbol{\alpha}, \mathbf{0}, F)\right\}$ could be considered, similarly as Huang et al. (2016) did under the Cox proportional hazards model. The development of the asymptotic properties of this test is an interesting topic of future research. The large sample properties for the estimation procedure that allows heterogeneity given in Web Appendix D will be studied in the future.

Although we synthesize the auxiliary information based on the non-mixture cure model, other cure models such as mixture cure models can be utilized to analyze cure rate survival data with the empirical likelihood approach. For clinical data, one can consider different types of data structures such as interval censored data. The covariates in our study are time-independent. The proposed estimation procedure may be extended to time-dependent covariates. Instead of utilizing auxiliary information in the form of survival probabilities, there may be other forms of auxiliary information. These problems deserve further research.

\section{ACKNowledgements}

The authors would like to thank the referee, the associate editor and the editor for their insightful comments that substantially improved this article. The research is partially supported by the National Natural Science Foundation of China [NSFC, Grant No. 11471065] and the China Scholarship Council [CSC, Grant No. 201906060019]. Ingrid Van Keilegom is financially supported by the European Research Council (2016-2021, Horizon 2020 / ERC grant agreement No. 694409). Part of the work on this article was completed during the first author's visit to KU Leuven. He would like to thank Ingrid Van Keilegom for her support. 
The data that support the findings of this study are available from the corresponding author upon request.

\section{REFERENCES}

Amico, M. and Van Keilegom, I. (2018). Cure models in survival analysis. Annual Review of Statistics and Its Application 5, 311-342.

Babu, G. J., Canty, A. J., and Chaubey, Y. P. (2002). Application of Bernstein polynomials for smooth estimation of a distribution and density function. Journal of Statistical Planning and Inference 105, 377-392.

Chatterjee, N., Chen, Y., Maas, P., and Carroll, R. (2016). Constrained maximum likelihood estimation for model calibration using summary-level information from external big data sources. Journal of the American Statistical Association 111, 107-117.

Chen, M., Ibrahim, J. G., and Sinha, D. (1999). A new Bayesian model for survival data with a surviving fraction. Journal of the American Statistical Association 94, 909-919.

Cox, D. (1972). Regression models and life tables (with discussion). Journal of the Royal Statistical Society, series B 34, 187-220.

Geerdens, C., Claeskens, G., and Janssen, P. (2013). Goodness-of-fit tests for the frailty distribution in proportional hazards models with shared frailty. Biostatistics 14, 433446.

Han, P. and Lawless, J. F. (2019). Empirical likelihood estimation using auxiliary summary information with different covariate distributions. Statistica Sinica 29, 1321-1342.

Hjort, N. L., McKeague, I. W., and Van Keilegom, I. (2018). Hybrid combinations of parametric and empirical likelihoods. Statistica Sinica 28, 2389.

Hu, T. and Xiang, L. (2013). Efficient estimation for semiparametric cure models with 
interval-censored data. Journal of Multivariate Analysis 121, 139-151.

Huang, C.-Y. and Qin, J. (2020). A unified approach for synthesizing population-level covariate effect information in semiparametric estimation with survival data. Statistics in Medicine 39, 1573-1590.

Huang, C.-Y., Qin, J., and Tsai, H. T. (2016). Efficient estimation of the Cox model with auxiliary subgroup survival information. Journal of the American Statistical Association 111, 787-799.

Hurvich, C. M. and Tsai, C.-L. (1989). Regression and time series model selection in small samples. Biometrika 76, 297-307.

Lorentz, G. G. (1986). Bernstein Polynomials. Chelsea Publishing Company, New York.

Maller, R. A. and Zhou, S. (1992). Estimating the proportion of immunes in a censored sample. Biometrika 79, 731-739.

Molanes-Lopez, E., Van Keilegom, I., and Veraverbeke, N. (2009). Empirical likelihood for non-smooth criterion functions. Scandinavian Journal of Statistics 36, 413-432.

Owen, A. B. (1988). Empirical likelihood ratio confidence intervals for a single functional. Biometrika 75, 237-249.

Portier, F., El Ghouch, A., and Van Keilegom, I. (2017). Efficiency and bootstrap in the promotion time cure model. Bernoulli 23, 3437-3468.

Qin, J. and Lawless, J. (1994). Empirical likelihood and general estimating equations. Annals of Statistics 22, 300-325.

Sheng, Y., Sun, Y., Deng, D., and Huang, C.-Y. (2020). Censored linear regression in the presence or absence of auxiliary survival information. Biometrics 76, 734-745.

Wang, J. and Ghosh, S. K. (2012). Shape restricted nonparametric regression with Bernstein polynomials. Computational Statistics and Data Analysis 56, 2729-2741.

Xue, H., Lam, K., and Li, G. (2004). Sieve maximum likelihood estimator for semipara- 
metric regression models with current status data. Journal of the American Statistical Association 99, 346-356.

Yakovlev, A. Y. and Tsodikov, A. D. (1996). Stochastic Models of Tumor Latency and Their Biostatistical Applications. World Scientific.

\section{SUPPORTING INFORMATION}

Web Appendices, Tables, and Figures referenced in Sections 4 to 6 are available with this paper at the Biometrics website on Wiley Online Library.

Received January 2020. Revised XXX 2020. Accepted XXX 2020. 


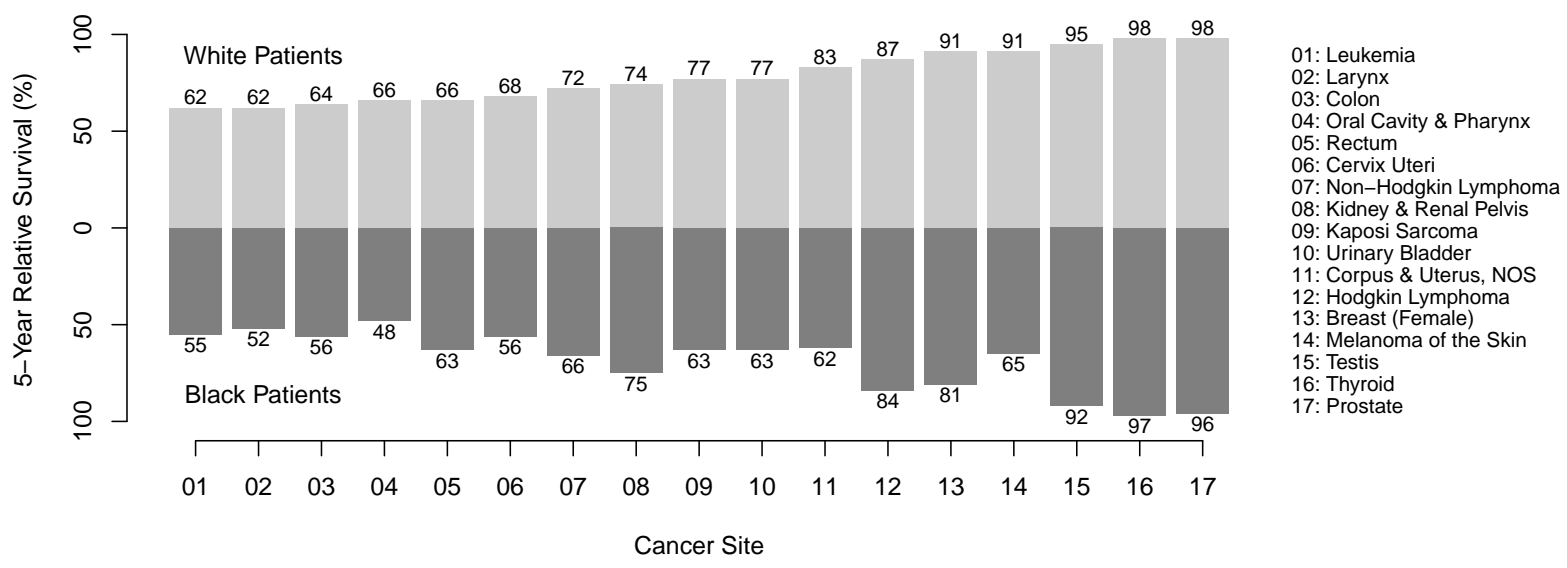

Figure 1: 5-year relative survival (\%), SEER program, 18 areas, 2008-2014, both sexes, by race and cancer site. 

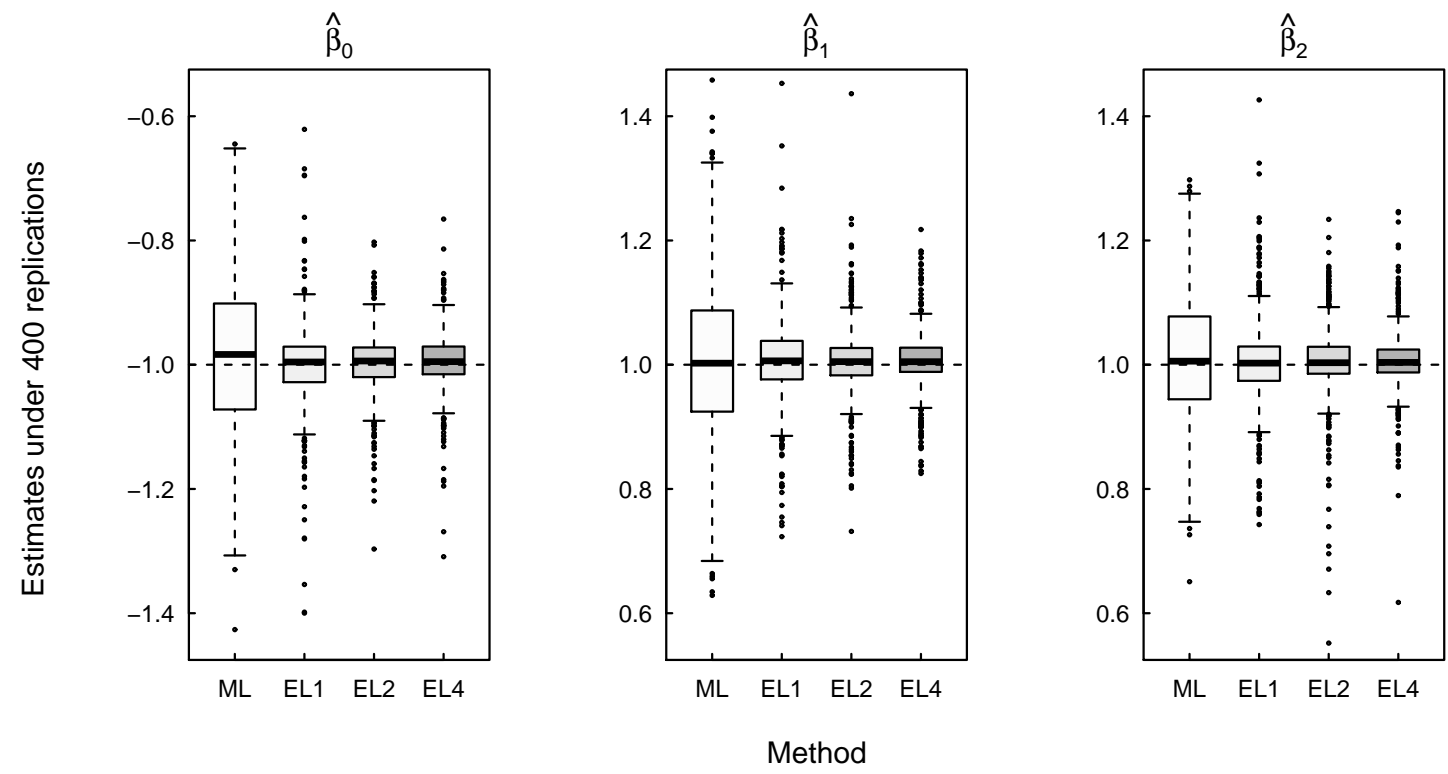

Figure 2: Boxplots of $400 \mathrm{ML}$ and EL estimates of $\left(\beta_{0}, \beta_{1}, \beta_{2}\right)$ under the moderate-level cure rate of Scenario I and $n=400$. 

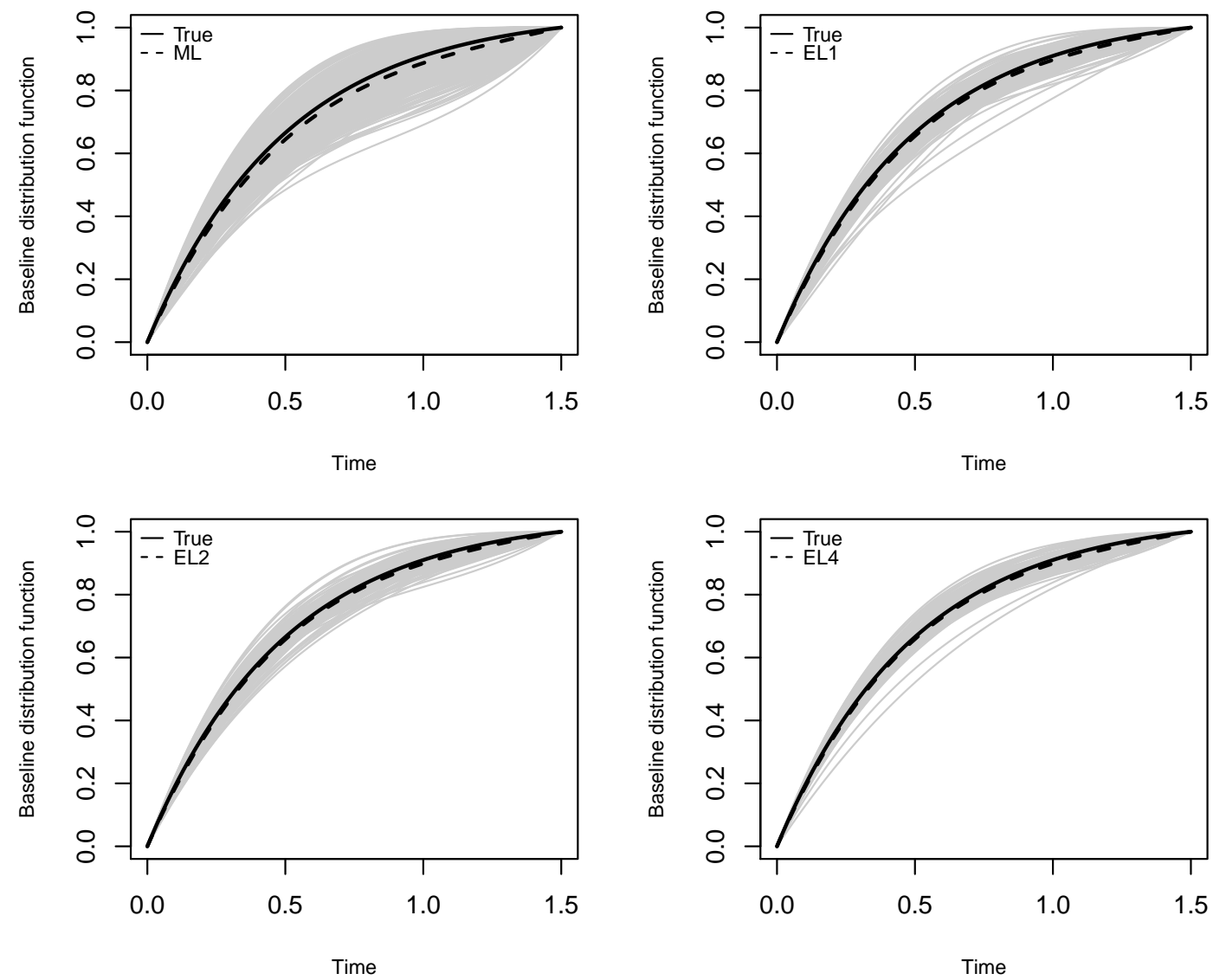

Figure 3: Curves of $400 \mathrm{ML}$ and EL estimates of the baseline distribution function under the moderate-level cure rate of Scenario I and $n=400$. The average of the 400 curves is given by the dashed curve, while the true baseline distribution function is represented by the solid curve. 
Table 1: Summarized simulation results under Scenario I for two cure rates.

\begin{tabular}{|c|c|c|c|c|c|c|c|c|c|c|c|c|}
\hline \multirow[b]{2}{*}{ Par } & \multirow[b]{2}{*}{$n$} & \multirow[b]{2}{*}{ Method } & \multicolumn{5}{|c|}{ Cure rate $15 \%$ (censoring rate $20 \%$ ) } & \multicolumn{5}{|c|}{ Cure rate $50 \%$ (censoring rate $60 \%$ ) } \\
\hline & & & Bias & $\mathrm{SD}$ & $\mathrm{SE}$ & $\mathrm{CP}$ & $\mathrm{RE}$ & Bias & $\mathrm{SD}$ & $\mathrm{SE}$ & $\mathrm{CP}$ & $\mathrm{RE}$ \\
\hline \multirow[t]{8}{*}{$\beta_{0}$} & \multirow[t]{4}{*}{200} & ML & 0.030 & 0.148 & 0.125 & 0.940 & - & -0.019 & 0.211 & 0.208 & 0.958 & - \\
\hline & & EL1 & 0.015 & 0.103 & 0.093 & 0.938 & 1.8 & 0.022 & 0.123 & 0.107 & 0.948 & 3.8 \\
\hline & & EL2 & -0.027 & 0.072 & 0.060 & 0.943 & 4.3 & -0.019 & 0.073 & 0.074 & 0.938 & 7.9 \\
\hline & & EL4 & -0.012 & 0.060 & 0.056 & 0.938 & 5.0 & -0.008 & 0.078 & 0.075 & 0.943 & 7.7 \\
\hline & \multirow[t]{4}{*}{400} & ML & -0.007 & 0.107 & 0.089 & 0.953 & - & -0.008 & 0.137 & 0.145 & 0.948 & - \\
\hline & & EL1 & -0.001 & 0.088 & 0.068 & 0.938 & 1.7 & 0.016 & 0.112 & 0.089 & 0.948 & 2.7 \\
\hline & & EL2 & -0.040 & 0.052 & 0.053 & 0.970 & 2.8 & 0.009 & 0.075 & 0.061 & 0.943 & 5.7 \\
\hline & & EL4 & -0.002 & 0.051 & 0.048 & 0.955 & 3.4 & 0.005 & 0.067 & 0.061 & 0.938 & 5.7 \\
\hline \multirow[t]{8}{*}{$\beta_{1}$} & \multirow[t]{4}{*}{200} & ML & 0.026 & 0.149 & 0.164 & 0.938 & - & 0.038 & 0.253 & 0.245 & 0.937 & - \\
\hline & & EL1 & 0.035 & 0.128 & 0.104 & 0.950 & 2.5 & -0.022 & 0.136 & 0.160 & 0.930 & 2.3 \\
\hline & & EL2 & -0.015 & 0.068 & 0.067 & 0.940 & 6.0 & 0.030 & 0.077 & 0.073 & 0.933 & 11.2 \\
\hline & & EL4 & -0.003 & 0.057 & 0.056 & 0.940 & 8.6 & 0.001 & 0.079 & 0.068 & 0.935 & 13.0 \\
\hline & \multirow[t]{4}{*}{400} & ML & 0.004 & 0.122 & 0.118 & 0.953 & - & 0.008 & 0.168 & 0.171 & 0.945 & - \\
\hline & & EL1 & -0.019 & 0.106 & 0.081 & 0.945 & 2.1 & -0.049 & 0.134 & 0.106 & 0.930 & 2.6 \\
\hline & & EL2 & -0.011 & 0.054 & 0.058 & 0.965 & 4.1 & -0.018 & 0.073 & 0.064 & 0.928 & 7.1 \\
\hline & & EL4 & -0.008 & 0.046 & 0.048 & 0.930 & 6.0 & -0.022 & 0.069 & 0.062 & 0.923 & 7.6 \\
\hline \multirow[t]{8}{*}{$\beta_{2}$} & \multirow[t]{4}{*}{200} & ML & 0.028 & 0.147 & 0.145 & 0.945 & - & 0.026 & 0.217 & 0.212 & 0.955 & - \\
\hline & & EL1 & 0.021 & 0.104 & 0.090 & 0.940 & 2.6 & -0.021 & 0.113 & 0.103 & 0.923 & 4.2 \\
\hline & & EL2 & -0.010 & 0.082 & 0.073 & 0.943 & 3.9 & 0.022 & 0.077 & 0.075 & 0.930 & 8.0 \\
\hline & & EL4 & -0.004 & 0.069 & 0.066 & 0.938 & 4.8 & 0.007 & 0.069 & 0.065 & 0.945 & 10.6 \\
\hline & \multirow[t]{4}{*}{400} & ML & 0.007 & 0.106 & 0.104 & 0.945 & - & 0.023 & 0.143 & 0.149 & 0.955 & - \\
\hline & & EL1 & -0.006 & 0.066 & 0.065 & 0.925 & 2.5 & 0.013 & 0.113 & 0.089 & 0.945 & 2.8 \\
\hline & & EL2 & 0.001 & 0.057 & 0.060 & 0.958 & 3.0 & 0.010 & 0.072 & 0.063 & 0.950 & 5.6 \\
\hline & & EL4 & 0.003 & 0.053 & 0.055 & 0.928 & 3.6 & 0.011 & 0.061 & 0.054 & 0.938 & 7.6 \\
\hline
\end{tabular}

NOTE: Bias, the empirical bias; SD, the standard deviation of 400 estimators; SE, the average of 400 estimated standard errors; $\mathrm{CP}$, the coverage probability of $95 \%$ confidence intervals; RE, the estimated variance under ML method divided by that under EL method; ML, the method of sieve maximum likelihood estimation without auxiliary information; EL1, the proposed estimation method with the auxiliary information at a single survival time $t^{*}$; EL2 and EL4, the same method with the auxiliary information at multiple time points $\left\{t_{1}^{*}, t_{2}^{*}\right\}$ and $\left\{t_{1}^{*}, \ldots, t_{4}^{*}\right\}$, respectively. 
Table 2: Summarized simulation results under Scenario II for two cure rates.

\begin{tabular}{|c|c|c|c|c|c|c|c|c|c|c|c|c|}
\hline \multirow[b]{2}{*}{ Par } & \multirow[b]{2}{*}{$n$} & \multirow[b]{2}{*}{ Method } & \multicolumn{5}{|c|}{ Cure rate $15 \%$ (censoring rate $25 \%$ ) } & \multicolumn{5}{|c|}{ Cure rate $50 \%$ (censoring rate $60 \%$ ) } \\
\hline & & & Bias & $\mathrm{SD}$ & $\mathrm{SE}$ & $\mathrm{CP}$ & $\mathrm{RE}$ & Bias & $\mathrm{SD}$ & $\mathrm{SE}$ & $\mathrm{CP}$ & $\mathrm{RE}$ \\
\hline \multirow[t]{8}{*}{$\overline{\beta_{0}}$} & \multirow[t]{4}{*}{200} & ML & -0.026 & 0.178 & 0.132 & 0.943 & - & -0.051 & 0.249 & 0.230 & 0.948 & - \\
\hline & & EL1 & 0.024 & 0.062 & 0.061 & 0.955 & 4.7 & 0.037 & 0.086 & 0.076 & 0.978 & 9.2 \\
\hline & & EL2 & -0.025 & 0.050 & 0.049 & 0.943 & 7.3 & 0.014 & 0.071 & 0.059 & 0.965 & 15.2 \\
\hline & & EL4 & -0.017 & 0.042 & 0.045 & 0.945 & 8.6 & 0.011 & 0.065 & 0.056 & 0.963 & 16.9 \\
\hline & \multirow[t]{4}{*}{400} & ML & -0.019 & 0.124 & 0.092 & 0.953 & - & -0.034 & 0.169 & 0.160 & 0.945 & - \\
\hline & & EL1 & 0.016 & 0.034 & 0.043 & 0.950 & 4.6 & 0.011 & 0.057 & 0.054 & 0.953 & 8.8 \\
\hline & & EL2 & 0.001 & 0.035 & 0.040 & 0.943 & 5.3 & -0.001 & 0.039 & 0.047 & 0.943 & 11.6 \\
\hline & & EL4 & 0.002 & 0.035 & 0.037 & 0.955 & 6.2 & -0.004 & 0.035 & 0.038 & 0.940 & 17.7 \\
\hline \multirow[t]{8}{*}{$\beta_{1}$} & \multirow[t]{4}{*}{200} & ML & 0.052 & 0.178 & 0.176 & 0.935 & - & 0.060 & 0.283 & 0.286 & 0.948 & \\
\hline & & EL1 & -0.025 & 0.064 & 0.067 & 0.940 & 6.9 & -0.040 & 0.117 & 0.080 & 0.973 & 12.8 \\
\hline & & EL2 & 0.007 & 0.042 & 0.049 & 0.948 & 12.9 & -0.030 & 0.051 & 0.054 & 0.933 & 28.1 \\
\hline & & EL4 & 0.007 & 0.044 & 0.043 & 0.945 & 16.8 & -0.023 & 0.050 & 0.046 & 0.948 & 38.7 \\
\hline & \multirow[t]{4}{*}{400} & ML & 0.028 & 0.129 & 0.123 & 0.937 & - & 0.042 & 0.195 & 0.199 & 0.950 & \\
\hline & & EL1 & -0.015 & 0.047 & 0.052 & 0.948 & 5.6 & -0.026 & 0.058 & 0.056 & 0.950 & 12.6 \\
\hline & & EL2 & -0.007 & 0.043 & 0.043 & 0.947 & 8.2 & -0.014 & 0.039 & 0.041 & 0.930 & 23.5 \\
\hline & & EL4 & -0.004 & 0.034 & 0.038 & 0.938 & 10.5 & -0.005 & 0.036 & 0.034 & 0.945 & 34.3 \\
\hline \multirow[t]{8}{*}{$\beta_{2}$} & \multirow[t]{4}{*}{200} & ML & 0.071 & 0.241 & 0.234 & 0.958 & - & 0.052 & 0.399 & 0.386 & 0.955 & \\
\hline & & EL1 & -0.030 & 0.057 & 0.066 & 0.938 & 12.6 & -0.046 & 0.089 & 0.077 & 0.975 & 25.1 \\
\hline & & EL2 & 0.018 & 0.048 & 0.048 & 0.930 & 23.8 & -0.034 & 0.077 & 0.053 & 0.975 & 53.0 \\
\hline & & EL4 & 0.014 & 0.045 & 0.044 & 0.928 & 28.2 & -0.027 & 0.044 & 0.044 & 0.940 & 76.9 \\
\hline & \multirow[t]{4}{*}{400} & ML & 0.045 & 0.167 & 0.160 & 0.937 & - & 0.049 & 0.273 & 0.266 & 0.945 & \\
\hline & & EL1 & -0.011 & 0.050 & 0.049 & 0.943 & 10.7 & -0.008 & 0.058 & 0.058 & 0.943 & 21.0 \\
\hline & & EL2 & 0.001 & 0.044 & 0.043 & 0.965 & 13.8 & -0.006 & 0.040 & 0.041 & 0.953 & 42.1 \\
\hline & & EL4 & -0.001 & 0.037 & 0.038 & 0.928 & 17.7 & 0.003 & 0.035 & 0.032 & 0.928 & 69.1 \\
\hline \multirow[t]{8}{*}{$\beta_{3}$} & \multirow[t]{4}{*}{200} & ML & -0.019 & 0.295 & 0.314 & 0.953 & - & 0.013 & 0.494 & 0.486 & 0.950 & \\
\hline & & EL1 & 0.019 & 0.067 & 0.079 & 0.950 & 15.8 & 0.029 & 0.069 & 0.080 & 0.955 & 36.9 \\
\hline & & EL2 & -0.006 & 0.045 & 0.048 & 0.955 & 42.8 & 0.020 & 0.052 & 0.050 & 0.955 & 94.5 \\
\hline & & EL4 & -0.006 & 0.048 & 0.040 & 0.963 & 61.6 & 0.017 & 0.040 & 0.039 & 0.948 & 155.3 \\
\hline & \multirow[t]{4}{*}{400} & ML & -0.009 & 0.206 & 0.218 & 0.945 & - & -0.012 & 0.341 & 0.336 & 0.945 & - \\
\hline & & EL1 & 0.006 & 0.057 & 0.056 & 0.958 & 15.2 & 0.013 & 0.064 & 0.058 & 0.958 & 33.6 \\
\hline & & EL2 & 0.005 & 0.044 & 0.042 & 0.953 & 26.9 & 0.011 & 0.035 & 0.040 & 0.930 & 70.5 \\
\hline & & EL4 & 0.002 & 0.035 & 0.034 & 0.945 & 41.1 & 0.005 & 0.030 & 0.029 & 0.940 & 134.2 \\
\hline
\end{tabular}

NOTE: the interpretations for Bias, SD, SE, CP, RE, ML and EL are the same as for Table 1. 
Table 3: Estimated regression coefficients, estimated standard errors and $p$-values for the melanoma study using the aggregate (complete) data and using the individual-level data based on the ML, EL1 and EL4 method.

\begin{tabular}{|c|c|c|c|c|c|c|}
\hline & \multicolumn{3}{|c|}{ Intercept } & \multicolumn{3}{|c|}{ Gender } \\
\hline & Est & $\mathrm{SE}$ & $p$-value & Est & $\mathrm{SE}$ & $p$-value \\
\hline Complete & -2.144 & 0.038 & 0 & 0.382 & 0.014 & $9 \mathrm{e}-168$ \\
\hline ML & -2.707 & 0.886 & 0.001 & 0.097 & 0.334 & 0.386 \\
\hline EL1 & -2.212 & 0.087 & $7 e-143$ & 0.361 & 0.077 & $1 \mathrm{e}-6$ \\
\hline \multirow[t]{3}{*}{ EL4 } & -2.256 & 0.043 & 0 & 0.328 & 0.036 & $2 \mathrm{e}-20$ \\
\hline & \multicolumn{3}{|c|}{ Age } & \multicolumn{3}{|c|}{ Tumor size } \\
\hline & Est & $\mathrm{SE}$ & $p$-value & Est & $\mathrm{SE}$ & $p$-value \\
\hline Complete & 1.570 & 0.028 & 0 & 0.640 & 0.026 & $1 \mathrm{e}-129$ \\
\hline ML & 1.217 & 0.720 & 0.045 & 0.309 & 0.561 & 0.291 \\
\hline EL1 & 1.509 & 0.088 & $1 \mathrm{e}-66$ & 0.579 & 0.083 & $2 \mathrm{e}-12$ \\
\hline EL4 & 1.456 & 0.051 & $5 e-178$ & 0.534 & 0.041 & $2 \mathrm{e}-38$ \\
\hline
\end{tabular}

